\title{
Lung Organoids and Their Use To Study Cell-Cell Interaction
}

\author{
Marko Z. Nikolić ${ }^{1}$ Emma L. Rawlins ${ }^{1}$
}

Published online: 24 April 2017

(C) The Author(s) 2017. This article is an open access publication

\begin{abstract}
Purpose of Review The lung research field has pioneered the use of organoids for the study of cell-cell interactions.

Recent Findings The use of organoids for airway basal cells is routine. However, the development of organoids for the other regions of the lung is still in its infancy. Such cultures usually rely on cell-cell interactions between the stem cells and a putative niche cell for their growth and differentiation.

Summary The use of co-culture organoid systems has facilitated the in vitro cultivation of previously inaccessible stem cell populations, providing a novel method for dissecting the molecular requirements of these cell-cell interactions. Future technology development will allow the growth of epithelialonly organoids in more defined media and also the introduction of specific non-epithelial cells for the study of cell interactions. These developments will require an improved understanding of the epithelial and non-epithelial cell types present in the lung and their lineage relationships.
\end{abstract}

Keywords Organoids $\cdot$ Lung progenitors $\cdot$ Mouse lung · Human lung $\cdot$ iPSCs

\section{Introduction}

Organoids are defined as three-dimensional (3D) structures derived from stem cells and consist of organ-specific cell

This article is part of the Topical Collection on Organoid Cultures

Emma L. Rawlins

e.rawlins@gurdon.cam.ac.uk

1 Gurdon Institute, University of Cambridge, Tennis Court Road, Cambridge CB2 1QN, UK types which self-organise through cell sorting and spatially restricted lineage commitment in a manner reminiscent of the native organ with some degree of organ functionality [1, 2]. Organoids have also been referred to as "mini-organs" and enable in vitro modelling of organ development, disease modelling and drug screening. The 3D culture preserves native DNA integrity and prevents the cells from being transformed [13]. Organoids were first successfully derived from mouse small intestine using single $\mathrm{Lgr}^{+}{ }^{+}$stem cells [3]. These organoids were entirely epithelial illustrating that organoids can be built without a non-epithelial cellular niche. The organoid was structured as crypt-villus units, with a similar stem cell hierarchy to in vivo, showing epithelial cell interactions are sufficient for the creation of crypt-villus units. Further work on cell-cell interactions using these intestinal organoids showed that essential niche signals are provided by Paneth cells, which are found interspersed between $\operatorname{Lgr} 5^{+}$ stem cells [4].

Organoid growth requires the initiating stem cell population to self-renew, to increase organoid size, and to differentiate. Organoids have been successfully cultured from multiple endoderm-derived organs including the adult mouse stomach [5], mouse colon [6], human colon [7], mouse pancreas [8], mouse liver [9], mouse prostate [10], human prostate [10], human intestine [11], and mouse embryonic pancreas [12]. In most of these studies, the same tissue culture medium supported both stem cell self-renewal and differentiation, for example intestinal stem cells self-organise efficiently into organoids and differentiate [3, 4]. By contrast, the adult liver and pancreas organoids can be expanded but do not differentiate easily yet $[13,14]$. Similarly, mouse embryonic pancreas progenitors were expanded in a self-renewing medium and then switched to a differentiation medium for maturation $[12,15,16]$. This switch in medium composition may be particularly important for organoids derived from embryonic 
progenitors as such cells are typically reliant on extrinsic signalling from the adjacent mesenchyme in vivo [17, 18]. Cellcell interactions within organoid cultures are likely to be just as important for differentiation as they are in vivo, through a process termed the "community effect". This is now a wellestablished phenomenon in which the differentiation ability of a cell is enhanced by neighbouring cells differentiating in the same way simultaneously [19].

Lung organoids have been successfully grown from the embryonic lungs [20-24], mouse adult lungs [25, 26, 27••, 28], human adult lungs [25, 28], and human iPSC (induced pluripotent stem cell) derived lung progenitors $[29,30,31 \bullet \cdot]$. This review will first introduce the lung as an organ with its various cell types and then evaluate the literature involving $3 \mathrm{D}$ cultures derived from lung stem cells, which we are referring to as organoids if they have demonstrated self-renewal capacity, or spheroids if self-renewal has not yet been achieved. We focus particularly on their currently widespread use for studying cell-cell interactions.

\section{The Lung and Its Cell Types}

Lung diseases account for the third highest mortality of noninfectious disease deaths and lung cancer is the most common cancer worldwide [32]. The mortality is partly due to irreversible destruction of lung tissue and the associated inability to meet the demands for lung transplantation. In order to address this, characterising the growth requirements of the different stem cell populations in both the adult and developing lungs

A Large human intra-lobar airway

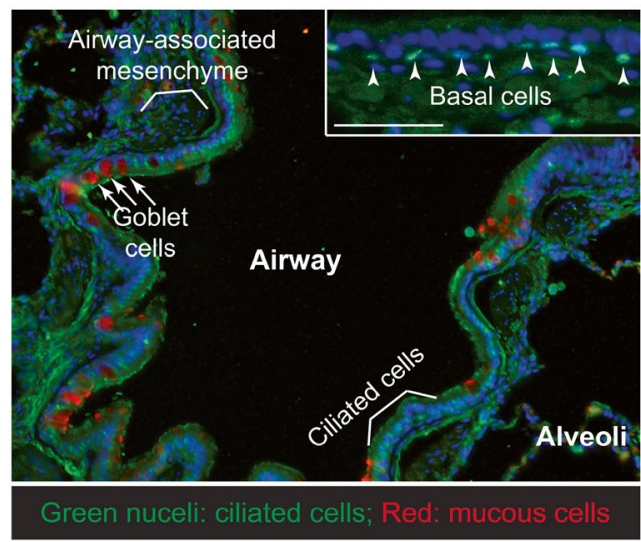

Fig. 1 Cellular organisation of the human lung. The cellular complexity of the human lung epithelium. a Section through large human intra-lobar airway stained to show ciliated cell nuclei $\left(\mathrm{FOXJ}^{+}\right.$; green) and mucousproducing Goblet cells (SCGB1A1 ${ }^{+} ;$red). It should be noted that Goblet cells are less prominent than Ciliated cells. The third major airway epithelial cell type (Basal cells) is shown in green in the inset as $\mathrm{TRP} 63^{+}$cells (arrowheads). The green background (due to autofluorescence from the tissue) nicely illustrates the airway-associated mesenchyme which has yet to be extensively characterised. b is essential. One approach is by studying cell-cell interactions in organoids.

The lung is a complex structure of branched epithelial-lined airways and endothelial-lined blood vessels which unite at the alveoli for gas exchange. The lung is surrounded by pleura; referred to as the mesothelium in mouse [33,34]. The trachea divides at the carina forming the left and right main stem bronchi. Each of which divides further into secondary, or lobar, bronchi and subsequently into progressively smaller bronchi and bronchioles, until the smallest bronchioles connect to the alveoli [35].

The human tracheobronchial airway is mostly lined by pseudostratified epithelium in which each cell type makes contact with the basement membrane. Below the basement membrane, blood vessels, smooth muscle, cartilage, extracellular matrix-producing fibroblasts, and nerves are found. The height of luminal epithelial cells and the proportion and density of the different cell types vary along the proximal-distal axis and between human and mouse [33]. The major airway epithelial cell types are basal, secretory (primarily goblet in human and club in mice), and ciliated cells, although neuroendocrine and brush cells form a more minor component (Fig. 1). Basal cells are present throughout human conducting airways but are confined to the trachea and primary bronchi of mice $[36,37]$.

The alveolar epithelium consists of type I and type II alveolar cells (AC1 and AC2) which are surrounded by capillaries and multiple different types of fibroblasts [38-40]. AC1 cells are flat, highly extended, and specialised for gas exchange as they cover more than $95 \%$ of the gas exchange surface area; whereas, AC2 cells are cuboidal, are more common, and are

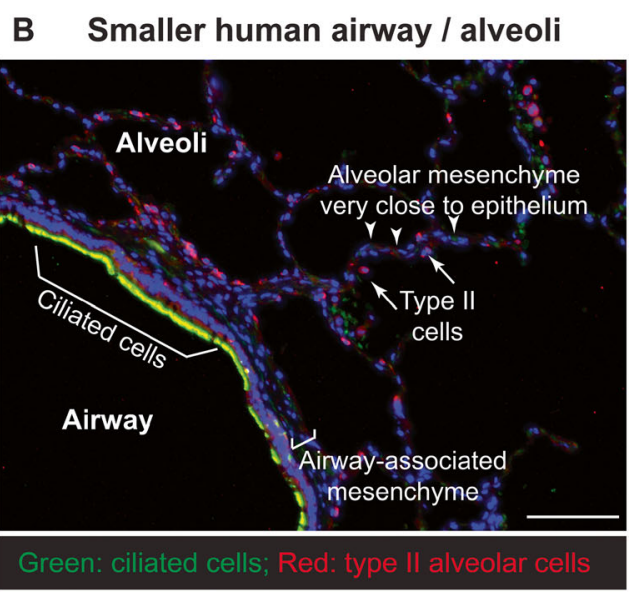

Section through a smaller human airway also showing the adjacent alveolar region. Here, cilia are visualised using an acetylated-tubulin antibody (green). Goblet cells are not shown, but are found at similar frequency to a. Note that the airway-associated mesenchyme is less extensive than in that in a. Type II alveolar cells are visualised using pro-SFTPC staining (red). The mesenchyme in the alveolar region is even less extensive and tightly associated with the epithelium. Scale bars: a $100 \mu \mathrm{m}$; b $50 \mu \mathrm{m}$ (inset) 
specialised for surfactant protein production [33, 38, 40, 41] (Fig. 1). Blood vessels are lined with vascular endothelial cells and also contain pericytes and vascular smooth muscle depending on size. The lymphatic system of the lung is poorly characterised and consists of lymphatic endothelial cells and associated stroma. The cellular interactions between alveolar macrophages, lung dendritic cells, and epithelial cells are thought to be crucial in restraining immune damage after infection or any other kind of damage to the epithelial barrier [42]. Controversy exists about the function of myofibroblasts and lipofibroblasts and other putative mesenchymal fibroblast populations. Their markers have not yet been defined, but they are thought to play important roles in lung alveolar development and maintenance [43].

The lung is a complex organ with numerous distinct cell types. How these cells interact in development, homeostasis, and disease is a key research question and organoids provide a platform for investigating some of these cell-cell interactions (Table 1).

\section{Organoids Derived from Embryonic Lungs}

In the mouse, lung development starts after establishment of the primary germ layers with the two primary endoderm buds becoming visible at around E9.5 [33]. Molecularly, the respiratory lineage can be first identified by expression of the transcription factor $\mathrm{Nkx} 2.1$ in the ventral anterior foregut endoderm [34, 47] as early as E8.25 [48]. The mouse lung then develops, similar to a human's, as a blind-ended tube which branches multiple times, a process governed by a complex network of transcription factors and signalling pathways. The important stem cell, or progenitor, population in the developing embryonic mouse lung is found in the distal branching tip [49]. These epithelial tip cells comprise a multipotent progenitor population giving rise to both bronchiolar and alveolar cells [49-51].

Whole E12.5 mouse lungs can be cultured in vitro on an air-liquid interface in 2D where they will both grow and differentiate [52-54]. These explant cultures could be described as the "ultimate mini-organs" as they contain all of the cells needed for normal lung development. However, their size, cellular complexity, and growth in a 2D plane, rather than in a $3 \mathrm{D}$, makes them unsuitable for many experiments.

Mixed cell populations obtained from dissociated whole E17.5 mouse lungs have been grown in 3D on various matrices [20-22]. Such cultures self-organise into spheroids containing branched epithelial structures surrounded by mesenchyme grow and show signs of alveolar and bronchiolar differentiation. However, self-renewal has not been tested and detailed cellular phenotyping has not been performed. These cultures have been used to test the effects of growth factors, such as FGFs and VEGF-A $[20,21]$, or for direct differentiation to alveolar structures [22]. Since whole foetal lungs were minced and then grown as spheroids, the culture is limited by the lack of a defined starting population. However, these spheroids could be useful as a tool for studying morphogenesis in vitro.

A recent advance is the formation of mesenchymal organoids consisting of human foetal lung (18- to 20-week gestation) fibroblasts grown on collagen-coated alginate beads in 3D units [23]. These were exposed to varying oxygen concentrations to mimic the oxygen tension experienced by premature neonates for disease modelling. Proof of concept experiments suggest that this system has the potential to incorporate additional lung cell types, including airway epithelial and endothelial cells [24]. It will be interesting to see if this bioengineering approach with defined cell types will be able to accurately recapitulate cell interactions and potentially even disease phenotypes.

\section{Organoids from iPSC-Derived Lung Epithelial Cells}

iPSCs are derived through reprogramming adult somatic cells by introducing pluripotency transcription factors [55]. The differentiation of patient-specific iPSCs into any relevant cell type can provide a platform for disease modelling, drug screening, and cell-based therapies. Several groups have successfully differentiated iPSCs into lung progenitors $[56,57]$ and more differentiated alveolar and bronchiolar lung epithelial cells [58-63]. All of these protocols used directed differentiation of iPSCs as monolayers in 2D culture systems and attempted to recapitulate normal development as described in the mouse lung developmental literature. Briefly, this involves differentiation into definitive endoderm, followed by anterior and ventral foregut endoderm. Ventral anterior foregut endoderm (VAFE) $\mathrm{Nkx} 2.1^{+}$lung progenitors are subsequently differentiated into bronchiolar or alveolar cell fates using a stage-specific combination of growth factors. Recent efforts have been made to increase the maturity of iPS-derived differentiated cells by 3D differentiation as organoids [31••, 64]. This approach has been successful to some extent for both alveolar [29] and bronchiolar differentiation, where there was evidence of effective differentiation into ciliated cells with beating cilia [30].

The first published attempt at deriving alveolar organoids from iPSC-produced human lung progenitors used carboxypeptidase $\mathrm{M}(\mathrm{CPM})$ as a cell-surface marker to specifically isolate VAFE iPSC-derived lung progenitors. These were then seeded into Matrigel with human foetal lung fibroblasts obtained at 17.5 weeks of gestation and an alveolar-specific combination of growth factors [29]. These NKX2.1 ${ }^{+}$ organoids contained small numbers of Aquaporin $5^{+} \mathrm{AC} 1$ cells and pro-SFTPC ${ }^{+}$(SPC) AC2 cells, the latter of which contained some structures reminiscent of lamellar bodies when analysed by transmission electron microscopy. 


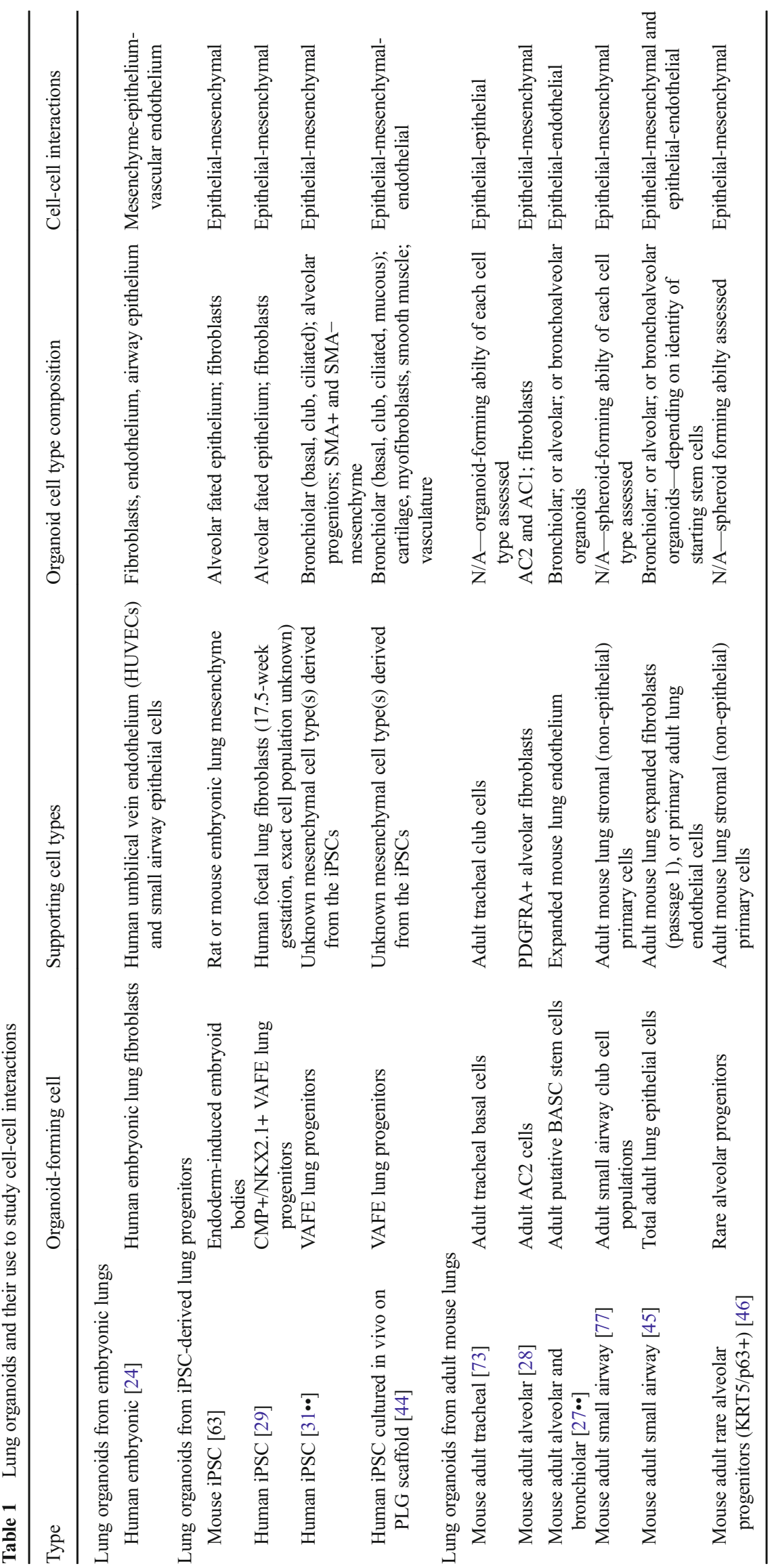


Interestingly, $\mathrm{CPM}^{+}$iPSC-derived lung progenitors cultured without human foetal lung fibroblasts were unable to produce $\mathrm{SPC}^{+}$cells, suggesting that the fibroblasts produce essential signal(s) for alveolar identity. Although the AC2-like cells were produced at low efficiency and are likely to be very immature, this co-culture is an encouraging strategy which could be improved upon by improved characterisation of the fibroblast cell type(s) and optimised culture conditions.

The same group used a similar strategy of isolating $\mathrm{CPM}^{+}$ iPSC-derived lung progenitors followed by organoid culture to produce bronchiolar lung organoids. In this case, there was evidence of differentiation into ciliated cells with beating cilia, as well as neuroendocrine, secretory, and basal cells [30]. Interestingly, there was no ciliated cell differentiation in $2 \mathrm{D}$ culture, suggesting that 3D organoid culture enabled successful differentiation into functional ciliated cells. However, ciliary beating was not fully synchronised as required for unidirectional flow and mucociliary clearance. This suggests functional immaturity, similar to the previous studies, or the lack of a directional cue. Organoids consisted only of epithelial cells without any evidence for mesenchymal differentiation.

Another study differentiated human pluripotent cells into VAFE spheroids [31••]. These foregut spheroids were then differentiated into lung organoids with both bronchiolar and alveolar regions and maintained in culture for over 3 months. Bronchiolar structures included basal, club, and ciliated cells and were often partially surrounded by smooth muscle actinexpressing and other uncharacterized, mesenchymal cells. The functional significance of the mesenchyme in these cultures has not yet been determined. Nevertheless, the proximity of epithelial and mesenchymal cells in these organoids may provide an in vitro platform to study the complex epithelialmesenchymal cell interactions in the developing embryonic lung. Alveolar structures in these organoids co-expressed HOPX and SOX9, or SPC and SOX9, consistent with early bipotent alveolar progenitors observed in mice, rather than differentiated alveolar cells [51, 65]. Transcriptome analysis of these human iPSC-derived lung organoids suggested that they are comparable to human foetal lung tissue despite longterm culture of over 3 months [31••]. The future study of epithelial-mesenchymal interactions will likely address this limitation. The same group has recently shown that xenotransplantation of human iPSC-derived lung organoids grown on poly(lactide-co-glycolide) (PLG) scaffolds into the kidney capsule of immunocompromised mice led to enhanced bronchiolar epithelial and mesenchymal organisation compared to organoids grown in vitro. Moreover, in vivo transplanted organoids had improved cellular differentiation of secretory and mesenchymal lineages and associated vasculature [44].

All of these studies illustrate that bronchiolar and alveolar iPSC-derived organoids can be grown in vitro to a degree of maturity which equates to foetal lung tissue. The most successful studies were those which produced organoids consisting of both epithelial and mesenchymal lineages, suggesting that epithelial-mesenchymal cell interaction is crucial for differentiation. Similar results have been found in the differentiation of other organs from human PSCs, including the kidney and intestine [66-68]. Further, maturation and validation by comparison to human adult tissue will be key to determining whether these lung organoids are suitable for future use in disease modelling, modelling of human lung development, and regenerative attempts. Similarly, human iPSCderived cardiomyocytes display a comparable degree of cellular immaturity (foetal phenotypes), despite 3D culture leading to higher levels of maturity than standard 2D culture. Approaches proposed for improving cardiomyocyte maturity include prolonging the culture period, high oxygen levels, and various combinations of either co-culture or growth factors [69]. Similar approaches could be used for lung organoids. The recent finding that in vivo transplanted lung organoids show improved signs of maturation is highly promising [44]. Although, one hypothesis that remains to be tested is whether the improved epithelial organisation depended on the mesenchyme differentiation or vice versa.

\section{Organoids Derived from the Adult Lungs}

Organoid culture has also been used to investigate stem cell identity and dissect cellular and molecular interactions for the adult lung. Single basal cells isolated from the adult mouse trachea have been grown into organoids, known as tracheospheres, in which the basal cells expand and, following a change in medium composition, differentiate [25]. These cultures could be passaged at least twice, demonstrating the basal cell's self-renewing ability, and formed part of the evidence that basal cells are adult stem cells. They have subsequently been used successfully to interrogate the roles of Notch, BMP, and IL6 signalling and specific transcription factors in adult basal cells $[26,70,71 \bullet, 72]$. Moreover, an elegant in vivo lineage-tracing strategy, combined with tracheosphere derivation, has been used to investigate basal-club cell interactions and demonstrate that in vitro basal cells are able to inhibit the de-differentiation of club cells [73]. The initial derivation of tracheospheres used media compositions that were optimised for expansion and differentiation of basal cells in 2D conditions at air-liquid interface [74]. These are still not completely defined and typically contain serum and bovine pituitary extract. More recently, attempts have been made to improve the growth conditions for basal cells in 2D which would subsequently facilitate organoid experiments. One approach has been to co-culture basal cells with fibroblast cells lines previously used for expansion of epidermis [75]. Another has been to inhibit SMAD signalling which apparently allows the expansion of basal cells from multiple organs, including the mouse and human trachea [76]. These publications raise interesting questions about the signalling interactions of airway basal cells in their in vivo niche which could 
be partly answered using organoids for co-culture experiments.

The smaller mouse airways consist of secretory club and ciliated cells. Attempts to grow club cells as spheroids rely exclusively on co-culture with underlying stromal populations. For example, isolated club cells could not be grown as spheres alone, only when co-cultured with an ill-defined fibroblast population [77]. Subsequent studies have used similar co-culture techniques and also highlighted the role of FGFR signalling, likely between the mesenchymal and epithelial cells in these co-cultures, demonstrating the utility of such organoid-based co-culture systems for analysing cell-cell signalling in vitro [45]. However, in all cases, the specific identity of the mesenchymal component of these co-cultures is lacking, making systematic analysis of the crosstalk difficult to achieve. By contrast, it has been definitively shown in vitro that co-culture as organoids with lung endothelial cells is sufficient to support the growth and differentiation of the putative BASC stem cells, epithelial cells located in the terminal bronchioles that co-express markers of bronchiolar and alveolar lineages $[27 \bullet \bullet]$. This co-culture system has been used to dissect a BMP4-based signalling interaction between the epithelial and endothelial cell types which is sufficient to promote alveolar linage differentiation. Multiple in vivo experiments suggest an intimate relationship between alveolar epithelial, endothelial, and haematopoietic cells, particularly for alveolar repair, which could be dissected in organoid-based systems [78-80].

Multiple in vivo and in vitro experiments have shown that, at steady state, the $\mathrm{AC} 2$ cells are the primary stem cell for the alveolar epithelium [28, 51]. Alveolar epithelial organoids, "alveolospheres", have been generated by co-culturing isolated AC2 cells with a PDGFRA ${ }^{+}$fibroblast population, which possibly corresponds to the currently ill-defined alveolar lipofibroblasts [28]. This organoid co-culture system has contributed to work showing that $\mathrm{AC} 2$ cells are more sensitive to decreases in telomere length than their supporting stromal population and that epithelial stem cell failure is the primary defect in lung diseases caused by telomere syndromes [81]. Further analysis of alveolar organoid co-culture systems will allow the signalling requirements for alveolar epithelial cell growth and differentiation to be determined.

An additional, less well-characterised, distal lung stem cell population contributes to mouse alveolar repair following severe injury, for example that caused by influenza infection. The steady-state characteristics of these cells are not thoroughly defined. However, they have been expanded in $2 \mathrm{D}$, there is consensus that they upregulate the basal cell markers keratin5 (KRT5) and p63 following injury, they are likely to be Sox2-positive, and there is some evidence for multilineage differentiation in organoid culture in vitro [82-85]. A recent publication has characterised a similar, probably identical or over-lapping, distal stem cell population as highly infected by influenza virus, capable of upregulating KRT5 and p63, and highly proliferative in spheroid culture when media was supplemented with FGF ligands, or in the presence of, currently uncharacterised, mesenchymal cells [46]. This is the first description of a spheroid co-culture system for the distal lung stem cell population and, although it has not yet been demonstrated to support self-renewal or differentiation, it is likely to prove highly useful for future mechanistic studies.

For disease modelling, generating mature disease-specific human lung cells is particularly important because murine models often do not completely phenocopy human lung disease. Methods for culturing human airway basal stem cells at air-liquid interface to recapitulate airway epithelial organisation are well-established [86] and have been adapted to include supporting cells, for example fibroblasts and vascular endothelium [87, 88]. Human airway basal cells are also highly amenable to growth and differentiation as organoids [25]. They can be efficiently genetically manipulated in organoid culture [71•], although attempts to further optimise these techniques remain ongoing in multiple labs. Culture of human AC2 cells as organoids has so far been less successful than that for mouse [28] and it is not yet possible to self-renew and differentiate human $\mathrm{AC} 2$ cells in $3 \mathrm{D}$ cultures.

\section{Conclusions: The Future of Lung Organoid Research}

Organoids have already proved extremely useful for lung research particularly for the study of adult mouse basal cells. In this case, they have provided an initial screening tool prior to pathway functional validation in vivo $[26,70,72]$ and also for working out mechanistic details in a simplified model system [73]. It is likely that the transition between using in vitro organoids and in vivo mouse work for different aspects of a project will increase in the future. The new ability to genetically modify organoids in vitro using modern gene-editing techniques, such as CRISPR-Cas9, will enable the functional analysis of genes involved in stem/progenitor self-renewal, differentiation, tissue morphogenesis, and even disease phenotypes [71•]. The rapid and ongoing application of organoid technologies to adult lung stem cells and lung development will undoubtedly facilitate human disease modelling and the more rapid cellular and molecular analysis of genetic variants that have so far only been identified as LOD scores in GWAS studies. Moreover, the development of using simple assays in intestinal organoids to study the response of individual cystic fibrosis patients to specific drugs [89] opens up the possibility of the development of similar assays for some of the highly heterogenous lung diseases, such as idiopathic pulmonary fibrosis. These 
exciting possibilities are leading to a renaissance in the study of human development and human disease.

One question which still needs to be answered is how complex do we need to make lung organoids for them to be useful? Throughout this review, we have highlighted the use of organoid technology for studying epithelial-epithelial and mesenchymal-epithelial cell interaction. But, should we be using these experiments to develop defined growth media which supports epithelial-only organoids? Or, should we be focusing on the inclusion of multiple cell types in a quest to more completely model the in vivo situation? The answers will most likely depend on the specific research question. However, we are particularly intrigued by the possibilities of using organoid technology to study the relationship between the epithelium and innate immune system or even to model the response to infection. Indeed, a recent report has used human intestinal organoids both as the first in vitro culture system for human norovirus and to study the epithelial response to infection [90]. A similar approach to study Pseudomonas infections in lung organoids that model aspects of cystic fibrosis, or bronchiectasis, may be highly fruitful.

The most significant limitations of current research using lung organoids are the lack of reliable validation by comparison to the in vivo cell types and the lack of defined starting populations. Particularly for human, differentiated organoids have been validated by marker expression from the mouse lung literature. An improved understanding of the numerous cell types in the developing and adult human lung is required to improve the validation and maturation attempts for lung organoids and will likely require more single-cell transcriptomics [65, 91]. In particular, a more reliable survey of the various mesenchymal lung cell types will facilitate the use of organoids for studying cell-cell interactions.

\section{Compliance with Ethical Standards}

Conflict of Interest The authors declare that they have no conflict of interest.

Human and Animal Rights and Informed Consent This article does not contain any studies with human or animal subjects performed by any of the authors.

Funding Sources Wellcome Trust PhD Programme for Clinicians to MZN, Medical Research Council G0900424 to ELR.

Open Access This article is distributed under the terms of the Creative Commons Attribution 4.0 International License (http:// creativecommons.org/licenses/by/4.0/), which permits unrestricted use, distribution, and reproduction in any medium, provided you give appropriate credit to the original author(s) and the source, provide a link to the Creative Commons license, and indicate if changes were made.

\section{References}

Recently published papers of particular interest have been highlighted as:

- Of importance

•- Of major importance

1. Nadkarni RR, Abed S, Draper JS (2016) Organoids as a model system for studying human lung development and disease. Biochem Biophys Res Commun 473:675-682. doi:10.1016/j.bbrc.2015.12.091

2. Clevers H (2016) Modeling development and disease with organoids. Cell 165:1586-1597. doi:10.1016/j.cell.2016.05.082

3. Sato T, Vries RG, Snippert HJ et al (2009) Single Lgr5 stem cells build crypt-villus structures in vitro without a mesenchymal niche. Nature 459:262-265. doi:10.1038/nature07935

4. Sato T, van Es JH, Snippert HJ et al (2011) Paneth cells constitute the niche for Lgr5 stem cells in intestinal crypts. Nature 469:415 418. doi:10.1038/nature 09637

5. Barker N, Huch M, Kujala P et al (2010) Lgr5+ve stem cells drive self-renewal in the stomach and build long-lived gastric units in vitro. Cell Stem Cell 6:25-36. doi:10.1016/j.stem.2009.11.013

6. Sato T, van Es JH, Snippert HJ et al (2011) Paneth cells constitute the niche for Lgr5 stem cells in intestinal crypts. Nature 469:415418. doi:10.1038/nature09637

7. Sato T, Stange DE, Ferrante M et al (2011) Long-term expansion of epithelial organoids from human Colon, adenoma, adenocarcinoma, and Barrett's epithelium. Gastroenterology 141:1762-1772. doi:10.1053/j.gastro.2011.07.050

8. Huch M, Bonfanti P, Boj SF et al (2013) Unlimited in vitro expansion of adult bi-potent pancreas progenitors through the Lgr5/Rspondin axis. EMBO J 32:2708-2721. doi:10.1038/emboj.2013. 204

9. Huch M, Dorrell C, Boj SF et al (2013) In vitro expansion of single Lgr5+ liver stem cells induced by Wnt-driven regeneration. Nature 494:247-250. doi:10.1038/nature11826

10. Karthaus WR, Iaquinta PJ, Drost J et al (2014) Identification of multipotent luminal progenitor cells in human prostate organoid cultures. Cell 159:163-175. doi:10.1016/j.cell.2014.08.017

11. Yin X, Farin HF, van Es JH et al (2014) Niche-independent highpurity cultures of Lgr5+ intestinal stem cells and their progeny. Nat Methods 11:106-112. doi:10.1038/nmeth.2737

12. Greggio C, De Franceschi F, Figueiredo-Larsen M, Grapin-Botton A. In vitro pancreas organogenesis from dispersed mouse embryonic progenitors. J Vis Exp. 2014:e51725-e51725. doi: 10.3791/ 51725

13. Huch M, Gehart H, van Boxtel R et al (2015) Long-term culture of genome-stable bipotent stem cells from adult human liver. Cell 160: 299-312. doi:10.1016/j.cell.2014.11.050

14. Broutier L, Andersson-Rolf A, Hindley CJ et al (2016) Culture and establishment of self-renewing human and mouse adult liver and pancreas 3D organoids and their genetic manipulation. Nat Protoc 11:1724-1743. doi:10.1038/nprot.2016.097

15. Greggio C, De Franceschi F, Figueiredo-Larsen M et al (2013) Artificial three-dimensional niches deconstruct pancreas development in vitro. Development 140:4452-4462. doi:10.1242/dev. 096628

16. Greggio C, De Franceschi F, Grapin-Botton A (2015) Concise reviews: in vitro-produced pancreas organogenesis models in three dimensions: self-organization from few stem cells or progenitors. Stem Cells 33:8-14. doi:10.1002/stem.1828

17. Laresgoiti U, Nikolić MZ, Rao C et al (2016) Lung epithelial tip progenitors integrate glucocorticoid and STAT3-mediated signals to control progeny fate. Development. doi:10.1242/dev.134023 
18. Bhushan A, Itoh N, Kato S et al (2001) Fgf10 is essential for maintaining the proliferative capacity of epithelial progenitor cells during early pancreatic organogenesis. Development 128:51095117

19. Gurdon JB (1988) A community effect in animal development. Nature 336:772-774. doi:10.1038/336772a0

20. Mondrinos MJ, Jones PL, Finck CM, Lelkes PI (2014) Engineering de novo assembly of fetal pulmonary organoids. Tissue Eng Part A 20:2892-2907. doi:10.1089/ten.TEA.2014.0085

21. Mondrinos MJ, Koutzaki S, Lelkes PI, Finck CM (2007) A tissueengineered model of fetal distal lung tissue. Am J Physiol Lung Cell Mol Physiol 293:L639-L650. doi:10.1152/ajplung.00403.2006

22. Zhang S, Zhou X, Chen T et al (2014) Single primary fetal lung cells generate alveolar structures in vitro. In Vitro Cell Dev Biol Anim 50:87-93. doi:10.1007/s11626-013-9657-6

23. Sucre JMS, Wilkinson D, Vijayaraj P et al (2016) A threedimensional human model of the fibroblast activation that accompanies bronchopulmonary dysplasia identifies Notch-mediated pathophysiology. Am J Physiol Lung Cell Mol Physiol 310: L889-L898. doi:10.1152/ajplung.00446.2015

24. Wilkinson DC, Alva-Ornelas JA, Sucre JMS et al (2016) Development of a three-dimensional bioengineering technology to generate lung tissue for personalized disease modeling. Stem Cells Transl Med. doi:10.5966/sctm.2016-0192

25. Rock JR, Onaitis MW, Rawlins EL et al (2009) Basal cells as stem cells of the mouse trachea and human airway epithelium. Proc Natl Acad Sci U S A 106:12771-12775. doi:10.1073/pnas.0906850106

26. Rock JR, Gao X, Xue Y et al (2011) Notch-dependent differentiation of adult airway basal stem cells. Cell Stem Cell 8:639-648. doi: 10.1016/j.stem.2011.04.003

27.• Lee J-H, Bhang DH, Beede A et al (2014) Lung stem cell differentiation in mice directed by endothelial cells via a BMP4-NFATc1thrombospondin-1 axis. Cell 156:440-455. doi:10.1016/j.cell.2013. 12.039 This paper uses a co-culture-based organoid system to dissect the detailed molecular interactions between a putative lung epithelial stem cell population and its endothelial cell niche

28. Barkauskas CE, Cronce MJ, Rackley CR et al (2013) Type 2 alveolar cells are stem cells in adult lung. J Clin Invest 123:3025-3036. doi:10.1172/JCI68782

29. Gotoh S, Ito I, Nagasaki T et al (2014) Generation of alveolar epithelial spheroids via isolated progenitor cells from human pluripotent stem cells. Stem Cell Reports 3:394-403. doi:10.1016/j. stemcr.2014.07.005

30. Konishi S, Gotoh S, Tateishi K et al (2016) Directed induction of functional multi-ciliated cells in proximal airway epithelial spheroids from human pluripotent stem cells. Stem Cell Reports 6:18 25. doi:10.1016/j.stemcr.2015.11.010

31.• Dye BR, Hill DR, Ferguson MAH et al (2015) In vitro generation of human pluripotent stem cell derived lung organoids. elife. doi:10. 7554/eLife.05098 Describes the development of lung organoids containing both bronchiolar and alveolar regions and associated mesenchyme derived from pluripotent stem cells

32. World Health Organization. Global status report on noncommunicable diseases 2010. 2011

33. Hogan BLM, Barkauskas CE, Chapman HA et al (2014) Repair and regeneration of the respiratory system: complexity, plasticity, and mechanisms of lung stem cell function. Cell Stem Cell 15:123138. doi:10.1016/j.stem.2014.07.012

34. Morrisey EE, Hogan BLM (2010) Preparing for the first breath: genetic and cellular mechanisms in lung development. Dev Cell 18:8-23. doi:10.1016/j.devcel.2009.12.010

35. Hacking C, Jones J. Bronchopulmonary segmental anatomy. In: 2016 httpradiopaedia.orgarticlesbronchopulmonary-segmentalanatomy. Accessed 28 Jul 2016

36. Boers JE, Ambergen AW, Thunnissen FB (1998) Number and proliferation of basal and parabasal cells in normal human airway epithelium. Am J Respir Crit Care Med 157:2000-2006. doi:10. 1164/ajrccm.157.6.9707011

37. Nakajima M, Kawanami O, Jin E et al (1998) Immunohistochemical and ultrastructural studies of basal cells, Clara cells and bronchiolar cuboidal cells in normal human airways. Pathol Int 48:944-953

38. Williams MC (2003) Alveolar type I cells: molecular phenotype and development. Annu Rev Physiol 65:669-695. doi:10.1146/ annurev.physiol.65.092101.142446

39. Herzog EL, Brody AR, Colby TV et al (2008) Knowns and unknowns of the alveolus. Proc Am Thorac Soc 5:778-782. doi:10. 1513/pats.200803-028HR

40. Weibel ER (2015) On the tricks alveolar epithelial cells play to make a good lung. Am J Respir Crit Care Med 191:504-513. doi: 10.1164/rccm.201409-1663OE

41. Crapo JD, Barry BE, Gehr P et al (1982) Cell number and cell characteristics of the normal human lung. Am Rev Respir Dis 126:332-337. doi:10.1164/arrd.1982.126.2.332

42. Kopf M, Schneider C, Nobs SP (2015) The development and function of lung-resident macrophages and dendritic cells. Nat Immunol 16:36-44. doi:10.1038/ni.3052

43. McCulley D, Wienhold M, Sun X (2015) The pulmonary mesenchyme directs lung development. Curr Opin Genet Dev 32:98-105. doi:10.1016/j.gde.2015.01.011

44. Dye BR, Dedhia PH, Miller AJ et al (2016) A bioengineered niche promotes in vivo engraftment and maturation of pluripotent stem cell derived human lung organoids. elife 5:e19732. doi:10.7554/ eLife.19732

45. Hegab AE, Arai D, Gao J et al (2015) Mimicking the niche of lung epithelial stem cells and characterization of several effectors of their in vitro behavior. Stem Cell Res 15:109-121. doi:10.1016/j.scr. 2015.05.005

46. Quantius J, Schmoldt C, Vazquez-Armendariz AI et al (2016) Influenza virus infects epithelial stem/progenitor cells of the distal lung: impact on Fgfr2b-driven epithelial repair. PLoS Pathog 12: e1005544. doi:10.1371/journal.ppat.1005544

47. Herriges M, Morrisey EE (2014) Lung development: orchestrating the generation and regeneration of a complex organ. Development 141:502-513. doi:10.1242/dev.098186

48. Serls AE, Doherty S, Parvatiyar P et al (2005) Different thresholds of fibroblast growth factors pattern the ventral foregut into liver and lung. Development 132:35-47. doi:10.1242/dev.01570

49. Rawlins EL, Clark CP, Xue Y, Hogan BLM (2009) The Id2+ distal tip lung epithelium contains individual multipotent embryonic progenitor cells. Development 136:3741-3745. doi:10.1242/dev. 037317

50. Alanis DM, Chang DR, Akiyama $\mathrm{H}$ et al (2014) Two nested developmental waves demarcate a compartment boundary in the mouse lung. Nat Commun 5:3923. doi:10.1038/ncomms4923

51. Desai TJ, Brownfield DG, Krasnow MA. Alveolar progenitor and stem cells in lung development, renewal and cancer. Nature. 2014: 1-16. doi: 10.1038/nature12930

52. del Moral P-M, Warburton D (2010) Explant culture of mouse embryonic whole lung, isolated epithelium, or mesenchyme under chemically defined conditions as a system to evaluate the molecular mechanism of branching morphogenesis and cellular differentiation. Methods Mol Biol 633:71-79. doi:10.1007/978-1-59745019-5 5

53. Seth R, Shum L, Wu F et al (1993) Role of epidermal growth factor expression in early mouse embryo lung branching morphogenesis in culture: antisense oligodeoxynucleotide inhibitory strategy. Dev Biol 158:555-559. doi:10.1006/dbio.1993.1213

54. Jaskoll TF, Don-Wheeler G, Johnson R, Slavkin HC (1988) Embryonic mouse lung morphogenesis and type II cytodifferentiation in serumless, chemically defined medium using prolonged in vitro cultures. Cell Differ 24:105-117 
55. Takahashi K, Yamanaka S (2006) Induction of pluripotent stem cells from mouse embryonic and adult fibroblast cultures by defined factors. Cell 126:663-676. doi:10.1016/j.cell.2006.07.024

56. Longmire TA, Ikonomou L, Hawkins F et al (2012) Efficient derivation of purified lung and thyroid progenitors from embryonic stem cells. Cell Stem Cell 10:398-411. doi:10.1016/j.stem.2012. 01.019

57. Mou H, Zhao R, Sherwood R et al (2012) Generation of multipotent lung and airway progenitors from mouse ESCs and patient-specific cystic fibrosis iPSCs. Cell Stem Cell 10:385-397. doi:10.1016/j. stem.2012.01.018

58. Firth AL, Dargitz CT, Qualls SJ et al (2014) Generation of multiciliated cells in functional airway epithelia from human induced pluripotent stem cells. Proc Natl Acad Sci U S A. doi:10. 1073/pnas.1403470111

59. Huang SXL, Islam MN, O'Neill J et al (2013) Efficient generation of lung and airway epithelial cells from human pluripotent stem cells. Nat Biotechnol. doi:10.1038/nbt.2754

60. Huang SXL, Green MD, de Carvalho AT et al (2015) The in vitro generation of lung and airway progenitor cells from human pluripotent stem cells. Nat Protoc 10:413-425. doi:10.1038/nprot.2015. 023

61. Wong AP, Bear CE, Chin S et al (2012) Directed differentiation of human pluripotent stem cells into mature airway epithelia expressing functional CFTR protein. Nat Biotechnol 30:876-882. doi:10. 1038/nbt.2328

62. Wong AP, Chin S, Xia S et al (2015) Efficient generation of functional CFTR-expressing airway epithelial cells from human pluripotent stem cells. Nat Protoc 10:363-381. doi:10.1038/nprot.2015. 021

63. Ghaedi M, Calle EA, Mendez JJ et al (2013) Human iPS cellderived alveolar epithelium repopulates lung extracellular matrix. J Clin Invest 123:4950-4962. doi:10.1172/JCI68793

64. Fox E, Shojaie S, Wang J et al (2015) Three-dimensional culture and FGF signaling drive differentiation of murine pluripotent cells to distal lung epithelial cells. Stem Cells Dev 24:21-35. doi:10. 1089/scd.2014.0227

65. Treutlein B, Brownfield DG, Wu AR et al (2014) Reconstructing lineage hierarchies of the distal lung epithelium using single-cell RNA-seq. Nature 509:371-375. doi:10.1038/nature13173

66. McCracken KW, Catá EM, Crawford CM et al (2014) Modelling human development and disease in pluripotent stem-cell-derived gastric organoids. Nature 516:400-404. doi:10.1038/nature13863

67. Watson CL, Mahe MM, Múnera J et al (2014) An in vivo model of human small intestine using pluripotent stem cells. Nat Med 20: 1310-1314. doi:10.1038/nm.3737

68. Takasato M, Er PX, Chiu HS et al (2016) Kidney organoids from human iPS cells contain multiple lineages and model human nephrogenesis. Nature 536:238-238. doi:10.1038/nature17982

69. Passier R, Orlova V, Mummery C (2016) Complex tissue and disease modeling using hiPSCs. Cell Stem Cell 18:309-321. doi:10. 1016/j.stem.2016.02.011

70. Tadokoro T, Gao X, Hong CC et al (2016) BMP signaling and cellular dynamics during regeneration of airway epithelium from basal progenitors. Development 143:764-773. doi:10.1242/dev. 126656

71. Gao X, Bali AS, Randell SH, Hogan BLM (2015) GRHL2 coordinates regeneration of a polarized mucociliary epithelium from basal stem cells. J Cell Biol 211:669-682. doi:10.1083/jcb.201506014 This publication elegantly applies gene editing technology to study differentiation of human airway basal cells grown as organoids

72. Tadokoro T, Wang Y, Barak LS et al (2014) IL-6/STAT3 promotes regeneration of airway ciliated cells from basal stem cells. Proc Natl Acad Sci U S A 111:E3641-E3649. doi:10.1073/pnas.1409781111
73. Tata PR, Mou H, Pardo-Saganta A et al (2013) Dedifferentiation of committed epithelial cells into stem cells in vivo. Nature 503:218 223. doi: $10.1038 /$ nature 12777

74. You Y, Richer EJ, Huang T, Brody SL (2002) Growth and differentiation of mouse tracheal epithelial cells: selection of a proliferative population. Am J Physiol Lung Cell Mol Physiol 283:L1315L1321. doi:10.1152/ajplung.00169.2002

75. Hynds RE, Butler CR, Janes SM, Giangreco A (2016) Expansion of human airway basal stem cells and their differentiation as 3D tracheospheres. Methods Mol Biol. doi:10.1007/7651 20165

76. Mou H, Vinarsky V, Tata PR et al (2016) Dual SMĀD signaling inhibition enables long-term expansion of diverse epithelial basal cells. Cell Stem Cell. doi:10.1016/j.stem.2016.05.012

77. Teisanu RM, Chen H, Matsumoto K et al (2011) Functional analysis of two distinct bronchiolar progenitors during lung injury and repair. Am J Respir Cell Mol Biol 44:794-803. doi:10.1165/rcmb. 2010-00980C

78. Ding B-S, Nolan DJ, Guo P et al (2011) Endothelial-derived angiocrine signals induce and sustain regenerative lung alveolarization. Cell 147:539-553. doi:10.1016/j.cell.2011.10.003

79. Rafii S, Cao Z, Lis R et al (2015) Platelet-derived SDF-1 primes the pulmonary capillary vascular niche to drive lung alveolar regeneration. Nat Cell Biol 17:123-136. doi:10.1038/ncb3096

80. Cao Z, Lis R, Ginsberg M et al (2016) Targeting of the pulmonary capillary vascular niche promotes lung alveolar repair and ameliorates fibrosis. Nat Med 22:154-162. doi:10.1038/nm.4035

81. Alder JK, Barkauskas CE, Limjunyawong $\mathrm{N}$ et al (2015) Telomere dysfunction causes alveolar stem cell failure. Proc Natl Acad Sci U S A 112:5099-5104. doi:10.1073/pnas.1504780112

82. Kumar PA, Hu Y, Yamamoto Y et al (2011) Distal airway stem cells yield alveoli in vitro and during lung regeneration following H1N1 influenza infection. Cell 147:525-538. doi:10.1016/j.cell.2011.10. 001

83. Vaughan AE, Brumwell AN, Xi Y et al (2015) Lineage-negative progenitors mobilize to regenerate lung epithelium after major injury. Nature 517:621-625. doi:10.1038/nature 14112

84. Zuo W, Zhang T, Wu DZ et al (2014) p63+Krt5+ distal airway stem cells are essential for lung regeneration. Nature 517:616-620. doi: 10.1038/nature 13903

85. Ray S, Chiba N, Yao C et al (2016) Rare SOX2(+) airway progenitor cells generate KRT5(+) cells that repopulate damaged alveolar parenchyma following influenza virus infection. Stem Cell Reports 7:817-825. doi:10.1016/j.stemcr.2016.09.010

86. Fulcher ML, Randell SH (2013) Human nasal and tracheobronchial respiratory epithelial cell culture. Methods Mol Biol 945:109-121. doi:10.1007/978-1-62703-125-7 8

87. Hill AR, Donaldson JE, Blume C et al (2016) IL- $1 \alpha$ mediates cellular cross-talk in the airway epithelial mesenchymal trophic unit. Tissue Barriers 4:e1206378. doi:10.1080/21688370.2016. 1206378

88. Curradi G, Walters MS, Ding B-S et al (2012) Airway basal cell vascular endothelial growth factor-mediated cross-talk regulates endothelial cell-dependent growth support of human airway basal cells. Cell Mol Life Sci 69:2217-2231. doi:10.1007/s00018-0120922-8

89. Dekkers JF, Wiegerinck CL, de Jonge HR et al (2013) A functional CFTR assay using primary cystic fibrosis intestinal organoids. Nat Med 19:939-945. doi:10.1038/nm.3201

90. Ettayebi K, Crawford SE, Murakami K et al (2016) Replication of human noroviruses in stem cell-derived human enteroids. Science 353:1387-1393. doi:10.1126/science.aaf5211

91. Xu Y, Mizuno T, Sridharan A et al (2016) Single-cell RNA sequencing identifies diverse roles of epithelial cells in idiopathic pulmonary fibrosis. JCI Insight 1:e90558. doi:10.1172/jci.insight. 90558 